\title{
Melanoma Growth Stimulatory Activity in Primary Malignant Melanoma: Prognostic Significance
}

Benton R. Middleman, M.D., Michael Friedman, M.D., David H. Lawson, M.D., Patricia B. DeRose, M.T., Cynthia Cohen, M.D.

Emory University School of Medicine, Atlanta, Georgia

Malignant melanoma (MM) cells do not require all exogenous growth factors of normal melanocytes. It is hypothesized that they make their own growth factors including melanoma growth stimulatory activity (MGSA). Cultured melanoma cells respond to MGSA with increased growth and angiogenesis suggesting a role for MGSA in MM proliferation, differentiation, and progression. We assessed the prognostic significance of MGSA expression in 37 primary MM immunostained for MGSA. Immunostains were graded for intensity $(0-3+)$, percentage of cells immunostained, and location of immunostain (intraepidermal, junctional, or dermal). In addition, 31 melanocytic and 23 dysplastic nevi were similarly studied for MGSA expression. All-MM showed the presence of immunostain, $6(16 \%) 1+$, $12(32 \%) 2+$, and $19(51 \%) 3+$. Six (16\%) had immunostain in $\leq 50 \%$ tumor cells, $31(84 \%)$ in $>50 \%$. A significant number of $\mathrm{MM}$ showed $>50 \%$ tumor cells staining at the dermal-epidermal junction compared with intraepidermal staining $(P<.0001)$. Intensity and amount of immunostain did not correlate with Clark's or Breslow's level. During a mean follow-up of 60 months (range: 5-101) on 27 patients, there were 4 local recurrences, 6 distant metastases, and 10 deaths. MGSA expression was not of prognostic significance with regard to survival (overall, disease free), or local recurrence or distant metastasis in primary MM. MGSA expression was similar in benign melanocytic and dysplastic nevi. Strong diffuse expression was noted in the junctional component of all junctional and most com-

Copyright (C) 2002 by The United States and Canadian Academy of Pathology, Inc.

VOL. 15, NO. 5, P. 532, 2002 Printed in the U.S.A.

Date of acceptance: January 8, 2002.

A support grant was received from the Melanoma Seed Grant Program of the Winship Center, Emory University Hospital, Atlanta, Georgia.

Presented, in part, at the United States Canadian Academy of Pathologists Annual Meeting, Toronto, Canada, March 1995 (in the Stowell Orbison Competition) and at the American Society of Clinical Pathologists/College of American Pathologists Spring Meeting, Boston, Massachusetts, April 1996.

Address reprint requests to: C. Cohen, M.D., Department of Anatomic Pathology, Emory University Hospital, G144, 1364 Clifton Road NE, Atlanta, GA 30322; e-mail: ccohe01@emory.edu; fax: 404-712-4754.

pound nevi. The dermal component consistently expressed less or no (in $45 \%$ of intradermal nevi) MGSA. MGSA expression does not correlate with prognosis in MM. Increased expression of MGSA at the dermal-epidermal junction in nevi and MM may indicate a role for MGSA in early local growth, before development of atypia.

KEY WORDS: Dysplastic nevus, Growth factors, Malignant melanoma, Melanocytic nevus.

Mod Pathol 2002;15(5):532-537

Numerous growth factors are produced by benign melanocytic nevi, dysplastic nevi, and primary and metastatic melanomas (1). In malignant melanoma (MM) cells, this is hypothesized to be the case because they do not require all exogenous growth factors, including melanoma growth stimulatory activity (MGSA), that normal melanocytes do (2). MGSA is one of a number of biologically active cytokines that have been localized to the skin (3). It is a mitogenic protein secreted by melanoma cell lines and corresponding to the polypeptide encoded by the human GRO gene (4). MGSA GRO $\alpha$ is a 73-amino acid peptide sharing sequence characteristics with the $\alpha$-chemokine superfamily. Three human MGSA/GRO genes encode three highly related chemokines, MGSA- $\alpha,-\beta$, and $-\gamma$. All bind to the same receptor with different affinities and stimulate chemotaxis, angiogenesis, and growth regulation $(5,6)$. The role of MGSA- $\alpha$ as an essential auto-stimulatory growth factor for melanoma cells is inhibited by preventing binding to its receptors (7). Cultured melanoma cells respond to MGSA with increased growth (8), suggesting that MGSA protein may potentially play a role in proliferation, differentiation, progression, and metastases of MM. Continuous expression of MGSA- $\alpha,-\beta$, or $-\gamma$ chemokine is suggested to contribute to transformation in mouse melanoma cell lines, resulting in increased ability to form large colonies in soft agar and tumors in nude mice (9). This MGSA-mediated transformation seems to require Ras activation (10). 
Several lesional steps have been described in tumor progression from nevus to $\mathrm{MM}(11,12)$ : (1) common acquired melanocytic nevus without architectural or cytologic atypia; (2) melanocytic nevus with persistent architectural atypia; (3) dysplastic nevus with persistent architectural atypia; (4) radial growth phase of primary MM with no competence for metastasis; (5) vertical growth phase of primary $\mathrm{MM}$ with competence to metastasize; and (6) metastatic MM. This multistep process is followed in $40-50 \%$ of superficial spreading melanomas, although some tumors first manifest at Step 3 or 4. In contrast, nodular MM appears to arise at Step 5. In this multistep process, growth usually precedes the development of architectural or cytological atypia. Therefore, altered production of growth factors or growth factor receptors might be suspected to occur early, before development of atypia.

To evaluate the hypothesis that MGSA production is altered early, before development of atypia, the frequency and site of expression of MGSA in benign and dysplastic nevi and in MM were studied. Results of MGSA expression in MM were related to thickness of tumor and to patient survival in an attempt to define its role in progression and prognosis of MM.

\section{MATERIALS AND METHODS}

Thirty-one consecutive melanocytic nevi, 23 dysplastic nevi, and 37 primary MM from the surgical pathology files at Emory University Hospital, Atlanta, Georgia, were studied. Only archival cases with available paraffin blocks containing sufficient tumor tissue were included. Nine melanocytic compound, 3 melanocytic junctional nevi, and 19 melanocytic intradermal nevi, 16 dysplastic compound, and 7 dysplastic junctional nevi, as well as 18 superficial spreading, 8 lentigo maligna, 6 nodular, and 5 acral lentiginous MM were studied. Follow-up information and prognostic parameters were obtained from the Oncology Data Bank of the Winship Cancer Center, Emory Clinic, Atlanta, Georgia, and by surgical pathology report and chart review.

Five-micrometer sections of formalin-fixed, paraffin-embedded tissue were examined for the presence of immunohistochemically detectable anti-human GRO- $\alpha$ (R and D Systems, Minneapolis, MN). An avidin-biotinylated enzyme complex kit (Bio Tek Solutions, Inc., Santa Barbara, CA) was used, with prior antigen retrieval and employing the automated TechMate 1000 immunostaining system (Bio Tek Solutions).

To prevent the presence of melanin from interfering with immunostain interpretation, all $\mathrm{MM}$ specimens were bleached before immunostaining.
This was achieved after deparaffinization and rehydration by sequential exposure to $0.25 \%$ potassium permanganate and $5 \%$ oxalic acid, then rinsing to clear the sections of melanin pigment.

The water rinse after bleaching the MM was replaced by buffer. All slides were loaded onto the TechMate, where they were exposed to hydrogen peroxide for 5 minutes, normal horse serum blocking reagent (1:20; Vector Laboratories, Inc., Burlingame, CA) for 5 minutes, primary antibody 1:40 for 25 minutes, biotinylated secondary linking antibody (Vector Laboratories) 1:100 for 25 minutes, avidin-biotinylated enzyme complex for $25 \mathrm{~min}$ utes, 3-amino-9-ethylcarbazole (AEC; Moss, Inc., Hanover, MD) as chromogen for 10 minutes, and hematoxylin as counterstain for 1 minute. These incubations were performed at room temperature; between incubations, sections were washed with buffer. At the end of the staining procedure, the slides were washed with distilled water and then coverslipped with Glycergel (DAKO Corporation, Carpinteria, CA), an aqueous, histologic mounting medium. They were not dehydrated through alcohol to xylene because alcohol dissolves the AEC color product.

Sections of MM tissue previously known to be positive for GRO- $\alpha$ (MGSA) were used as positive controls; the antibody was replaced by buffer in negative controls. Immunostained slides were reviewed by two pathologists (BM, CC) and assessed, by consensus, according to the intensity of immunostain graded as $1+$ to $3+$. The percentage of tumor cells staining was recorded as negative, $\leq 50 \%$, or $>50 \%$, and the location of the stain was noted (intraepidermal, junctional, or intradermal), the latter as radial- and/or vertical-growth phase in MM.

Statistics were performed using the Fisher exact test. Kaplan-Meier survival curves were constructed comparing survival of MM patients with and without MGSA expression in the primary MM. A $P$ value of $<.05$ was considered significant.

\section{RESULTS}

The 31 melanocytic nevi were from 23 patients ( 4 male, 19 female), with a mean age of 32 years (range, 2-75 y). The 23 dysplastic nevi were from 17 patients (10 male, 7 female), with a mean age of 42 years (range, 26-69 y). Of the $37 \mathrm{MM}, 23$ patients were female and 14 male, with a mean age of 59 years (range, 27-91 y). Seventeen MM were on the extremities, 11 in the head and neck region, and 9 on the trunk. Clark's levels were 10 Level I, 15 Level II, 3 Level III, 6 Level IV, and 3 Level V; Breslow's depth of invasion was $10 \mathrm{in} \mathrm{situ;} \mathrm{14,} \mathrm{<0.76;} \mathrm{3,} \mathrm{0.76-}$ 1.49 ; $1,1.50-2.49 ; 5,2.50-3.99$; and $4, \geq 4.00 \mathrm{~mm}$. 


\begin{tabular}{|c|c|c|c|c|c|c|c|}
\hline \multirow[t]{2}{*}{ Type of Melanocytic Nevi } & \multirow[t]{2}{*}{ Total } & \multicolumn{4}{|c|}{ Staining Intensity } & \multicolumn{2}{|c|}{$\begin{array}{c}\% \text { Cells } \\
\text { Immunostained }\end{array}$} \\
\hline & & 0 & 1 & 2 & 3 & $\leq 50$ & $>50$ \\
\hline \multicolumn{8}{|l|}{ Melanocytic NEVI (31) } \\
\hline Compound (junctional component) & 9 & 0 & 1 & 2 & 6 & 1 & 8 \\
\hline Compound (dermal component) & 9 & 4 & 0 & 2 & 3 & 8 & 1 \\
\hline Junctional & 3 & 0 & 0 & 1 & 2 & 0 & 3 \\
\hline Intradermal nevi & 19 & 9 & 0 & 6 & 4 & 17 & 2 \\
\hline Total & 31 & & & & & 25 & 14 \\
\hline \multicolumn{8}{|l|}{ Dysplastic NEVI (23) } \\
\hline Compound (junctional component) & 16 & 0 & 0 & 8 & 8 & 8 & 8 \\
\hline Compound (dermal component) & 16 & 3 & 2 & 11 & 0 & 11 & 5 \\
\hline Junctional nevi & 7 & 0 & 0 & 0 & 7 & 0 & 7 \\
\hline Total & 23 & & & & & 19 & 20 \\
\hline
\end{tabular}

MGSA, melanoma growth stimulatory activity.

Table 1 shows the staining intensity and percentage of cells immunostained for MSGA according to type of nevus and location of the cells examined. All 10 cases of junctional nevi ( 3 melanocytic and 7 dysplastic) showed intense $(2-3+)$ and diffuse $(>50 \%)$ immunostain. Intense $(2-3+)$ immunostain was seen at the epidermal-dermal junction (junctional component) of 8 (89\%) compound benign nevi (Fig. 1 ) and $16(100 \%)$ compound dysplastic nevi. The junctional component of compound nevi (melanocytic and dysplastic) showed diffuse $(>50 \%)$ staining in $8(89 \%)$ and $8(50 \%)$, respectively.

The dermal component of the compound lesions consistently tended to express less MGSA than the junctional component (Fig. 1). Four (44\%) compound melanocytic nevi shôwed no immunostain in their dermal component. Three cases (33\%) showed $3+$ immunostain, whereas $2(22 \%)$ showed $2+$ staining. In only $1(11 \%)$ case did the dermal component of the compound melanocytic nevus stain diffusely ( $>50 \%$ ) for MGSA. The dermal component of compound dysplastic nevi expressed no MGSA in $3(19 \%)$ cases, $1+$ staining in $2(13 \%)$

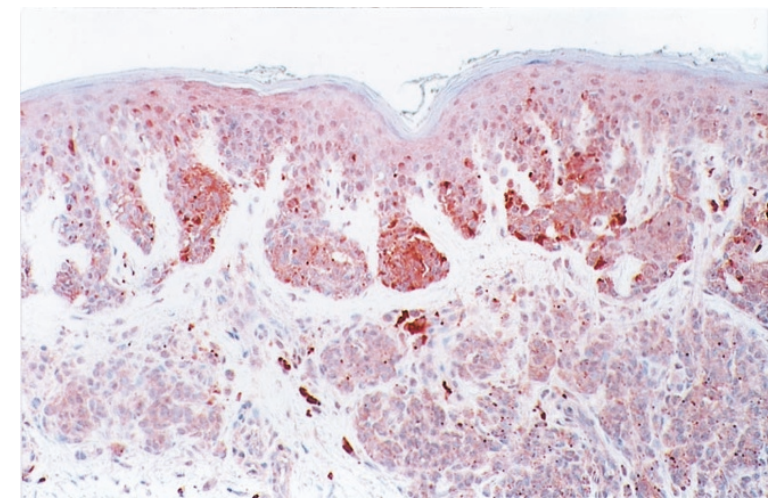

FIGURE 1. Strong $(3+)$ diffuse $(>50 \%)$ melanoma growth stimulatory activity (MGSA/GRO- $\alpha$ ) expression in the junctional component of a compound melanocytic nevus as compared with the weaker $(1-2+)$ expression in the dermal component. Histiocytes are 3+ immunoreactive; magnification, $40 \times$. cases, and $2+$ staining in $11(68 \%)$ cases. Staining intensity of $3+$ was not seen in the dermal component of any compound dysplastic nevus. In only 5 $(31 \%)$ cases did the dermal component of compound dysplastic nevi stain diffusely $(>50 \%$ of cells). Of the 19 intradermal nevi, 9 (47\%) did not stain for MGSA, and only $2(11 \%)$ showed stain in $>50 \%$ of the cells.

All MM showed the presence of immunostain (Table 2). Six (16\%) were graded $1+, 12(32 \%)$ were graded $2+$, and $19(51 \%)$ were graded $3+$. Six $(16 \%)$ had immunostain in $\leq 50 \%$ of tumor cells, and 31 $(84 \%)$ had immunostain in $>50 \%$ of tumor cells. Thus, $83 \%$ of MM graded $2-3+$ and $84 \%$ had immunostain present in $>50 \%$ of tumor cells (Figs. 2, 3).

Clinical correlation was available on $27 \mathrm{MM}$ patients followed from 5-101 months (mean, $60 \mathrm{mo}$ ). Table 2 shows patient status at follow-up. Seventeen patients are alive, 13 of whom remained free of recurrence or metastasis during the follow-up period. Three patients had local recurrence of their MM that was subsequently re-excised. One patient is currently alive with unresectable tumor. Ten patients died during the follow-up period. Five (50\%) died secondary to $\mathrm{MM}$, and 5 (50\%) from an inter-

TABLE 2. Intensity and Amount of MGSA in 37 Malignant Melanomas, Related to Patient Follow-Up Status in 27

\begin{tabular}{|c|c|c|c|c|c|}
\hline \multirow{2}{*}{ Patient Status } & \multirow{2}{*}{$1+$} & \multirow{2}{*}{$2+$} & \multirow{2}{*}{$3+$} & \multirow{2}{*}{$\frac{\text { Immunostain }}{\leq 50 \%}$} & \multirow{2}{*}{$\frac{\text { Amount }}{>50 \%}$} \\
\hline & & & & & \\
\hline \multicolumn{6}{|l|}{ Alive (17) } \\
\hline Free of disease (13) & 1 & 4 & 8 & 1 & 12 \\
\hline Recurred, re-excised (3) & & 2 & 1 & 1 & 2 \\
\hline Alive with disease (1) & & & 1 & & 1 \\
\hline \multicolumn{6}{|l|}{ Dead (10) } \\
\hline Secondary to melanoma (5) & 1 & 2 & 2 & 1 & 4 \\
\hline Intercurrent disease (5) & 2 & 1 & 2 & 3 & 2 \\
\hline Unknown (10) & 2 & 3 & 5 & & 10 \\
\hline Total (37) & 6 & 12 & 19 & 6 & 31 \\
\hline
\end{tabular}

MGSA, melanoma growth stimulatory activity. 


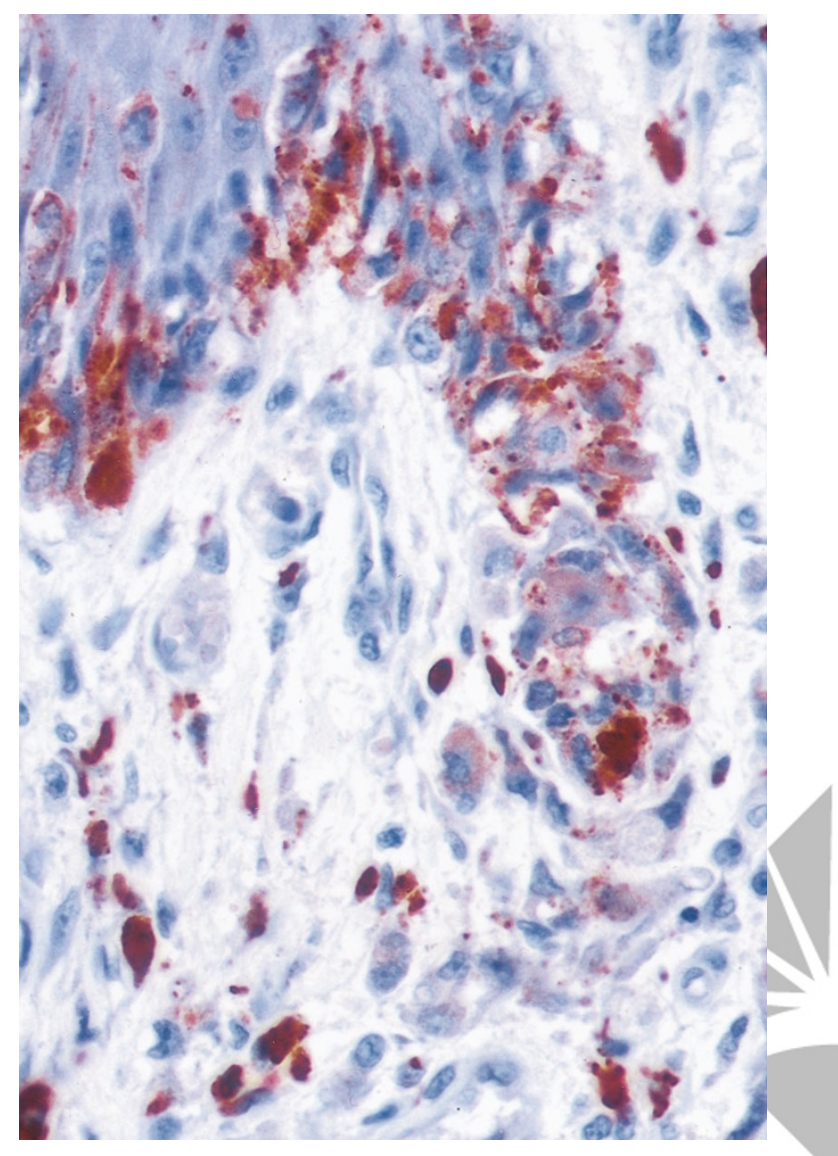

FIGURE 2. Diffuse $(>50 \%)$ and intense $(3+)$ cytoplasmic MGSA/ GRO- $\alpha$ immunostaining of malignant melanoma cells at the epidermaldermal junction. Histiocytes within the dermis also show staining; magnification, $400 \times$.

TABLE 3. Survival in 27 Malignānt Melanoma Patients Related to Intensity and Amount of Immunostain for MGSA ount of immunostain for

\begin{tabular}{lccccc}
\hline & \multicolumn{4}{c}{ Immunostain } \\
\cline { 2 - 5 } & \multicolumn{2}{c}{ Intensity } & Amount \\
\hline Survival & $1+$ & $2+$ & $3+$ & $\leq 50 \%$ & $>50 \%$ \\
Mean overall survival (months) & 34 & 66 & 65 & 68 & 56 \\
Mean disease-free survival (months) & 34 & 59 & 61 & 68 & 51 \\
\hline
\end{tabular}

MGSA, melanoma growth stimulatory activity.

\section{Prohilent}

TABLE 4. Intensity and Amount of MGSA Immunostain in 27* Malignant Melanoma Related to Breslow Depth of Invasion

\begin{tabular}{lcccccc}
\hline Breslow (MM) & $1+$ & $2+$ & $3+$ & $\leq 50 \%$ & $>50 \%$ & TOTAL \\
\hline$<0.76$ & 1 & 7 & 6 & 3 & 11 & 14 \\
$0.76-1.49$ & 1 & 1 & 1 & 0 & 3 & 3 \\
$1.50-2.49$ & 1 & 0 & 0 & 0 & 1 & 1 \\
$2.50-3.99$ & 0 & 1 & 4 & 1 & 4 & 5 \\
$>4.00$ & 1 & 0 & 3 & 0 & 4 & 4 \\
Total & 4 & 9 & 14 & 4 & 23 & $27^{*}$ \\
\hline
\end{tabular}

* 10 in situ MM were not included.

MGSA, melanoma growth stimulatory activity.

Only 8 (35\%) of 33 lesions showed $>50 \%$ of cells staining in the intraepidermal portion of the lesion. A significant number of MM showed $>50 \%$ of tumor cells staining at the dermal-epidermal junction (Fig. 3) when compared with intraepidermal staining $(P<.0001$; Table 5$)$. There were, however, no significant differences in the intensity or percentage immunostain between the intraepidermal or junctional, and intradermal components of the MM studied.

No difference in staining intensity or percentage of cells stained was detected among the types of MM. Specifically, $100 \%(6 / 6)$ of the nodular and $78 \%(14 / 18)$ of the superficial spreading $\mathrm{MM}$ stained with $2-3+$ intensity. Sixty-seven percent (4/6) of the nodular MM and $89 \%(16 / 18)$ of the superficial spreading MM showed staining in $>50 \%$ of the tumor cells $(P=.25)$. Also, no significant difference in staining intensity or percentage of cells immunostained wăs found when comparing MM from the different anatomical locations.

\section{DISCUSSION}

We have demonstrated the presence of MGSA in all the benign melanocytic and dysplastic nevi and all the primary MM studied. No significant differences in MGSA expression were noted between be- current disease process. Ten patients were lost to follow-up. Intensity and percentage immunostain did not correlate with survival when comparing those patients alive and free of disease with patients who died secondary to MM (Table 3 ).

Intensity and percentage immunostain did not correlate with depth of the lesion (in millimeters; Breslow depth of invasion; Table 4). Lesions of $<0.76 \mathrm{~mm}$, when compared with lesions of $>1.5$ $\mathrm{mm}$ in thickness, revealed no significant difference in staining intensity $(P=.55)$ or percentage cells immunostained $(P=.61)$. Likewise, the stated staining criteria did not correlate with increasing Clark level.

Twenty-seven (73\%) MM showed $>50 \%$ of tumor cells staining in the junctional location (Table 5).

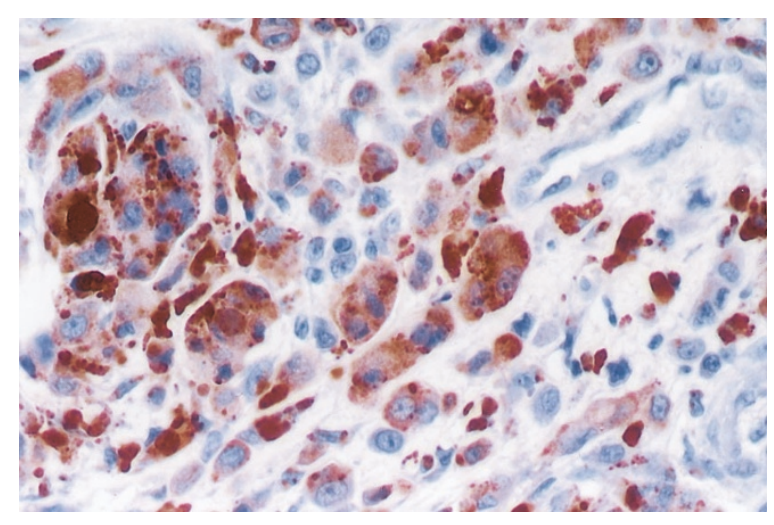

FIGURE 3. Strong $(3+)$ and diffuse $(>50 \%)$ cytoplasmic MGSA immunostain in vertical-growth phase malignant melanoma cells and in histiocytes; magnification, $400 \times$. 
TABLE 5. Intensity and Amount of MGSA Immunostain According to Location in $\mathbf{3 7}$ Malignant Melanomas

\begin{tabular}{lrrrcrc}
\hline \multicolumn{1}{c}{ Location } & $1+$ & $2+$ & $3+$ & $\leq 50 \%$ & $>50 \%$ & N/A \\
\hline Intraepidermal (IE) & 8 & 11 & 14 & 25 & 8 & 4 \\
Junctional (J) & 6 & 15 & 16 & 10 & 27 & 0 \\
Intradermal (ID) & 4 & 5 & 8 & 6 & 11 & 20 \\
\hline
\end{tabular}

MGSA, melanoma growth stimulatory activity; N/A, Not available; IE versus $\mathrm{J}(\mathrm{P}=.0001)$; J versus ID $(\mathrm{P}=.54)$; IE versus $\mathrm{ID}(\mathrm{P}=.38)$.

nign melanocytic and dysplastic nevi. Strong and diffuse ( $>50 \%$ ) MGSA expression was present in the junctional component of all junctional nevi and the majority of compound nevi. The dermal component in the latter consistently expressed less, whereas almost half the intradermal nevi failed to express MGSA. A majority of MM showed strong and diffuse expression of MGSA, significantly greater at the dermal-epidermal junction at which proliferation occurs than in the intraepidermal component of the lesions $(P=.0001)$, but similar to that in the intradermal component. Our data suggest that MGSA is expressed as an early marker of local growth in nevi and MM and of tumor progression in the intradermal component of MM. MGSA appears to have an insignificant predictive role in the identification of the ability of MM to metastasize, because fairly large amounts were present in all MM and in all components of each MM. We also failed to show prognostic significance because MGSA expression was unrelated to depth of invasion, metastatic potential, and patient survival. MGSA has also been shown not to be useful as a marker for diagnosis of MM, being only $57 \%$ sensitive and $40 \%$ specific for MM (13).

Existing data suggest that the MGSA protein may potentially play a role in the proliferation, differentiation, progression, and metastases of MM. Cells of dysplastic nevi, advanced primary melanoma, and metastatic melanoma require different growth factors for proliferation in vitro (11). MGSA mRNA is constitutively expressed in the absence of exogenous growth factors in cultures established from benign intradermal and dysplastic nevi and melanoma lesions in different stages of tumor progression (1). Purified MGSA, initially identified as a factor secreted by the human melanoma cell line Hs294T, was found to have the ability to mitogenically stimulate these same melanoma cells (8). Later, purified recombinant MGSA was found to be mitogenically active on melanoma cells in vitro (4).

MGSA/GRO protein expression has been identified in a number of benign and malignant lesions. In fixed sections, Tettelbach et al. (14) report one of three congenital nevi, three of four compound nevi, and five of five dysplastic nevi to be MGSA/GRO positive. Richmond and Thomas (15) found 19 of 27 (70\%) melanoma, 7 of 32 (22\%) nevus cultures, and $75 \%$ of 15 neval sections to be MGSA positive. In 31 benign and 23 dysplastic nevi, we have found MGSA expression in all junctional nevi and at the junction of the majority of compound (benign and dysplastic) nevi. In contrast, almost half of the dermal nevi did not stain for MGSA, and dermal expression was consistently less than junctional expression in compound lesions. These findings support existing data of high proliferating cell nuclear antigen (PCNA), a marker of proliferation, in nevus cells at the dermal-epidermal junction and in the papillary dermis but virtually no PCNApositive nevus cells in the deeper dermis (16). Benign congenital acquired and dysplastic nevi have been found to display no or very low intradermal melanocytic proliferation determined by MIB-1 immunoreactivity (17). Rudolph et al. (18), assessing growth fraction with two monoclonal antibodies (Ki-S1 and Ki-55), found it to be constantly higher in the epidermal-junctional compartment than in the dermal aggregations of compound nevi. Basic fibroblast growth factor has been identified in the basement membranes at the dermal-epidermal junction and surrounding nevus cell nests and individual cells at that site (19). These findings suggest a role for MGSA as a local growth factor in proliferation of acquired melanocytic nevi.

Data suggest that protein encoded by MGSA/ GRO mRNA may play a role in inflammation and exert its effect on endothelial cells in an autocrine fashion (20). Human umbilical vein endothelial cells are capable of synthesizing and secreting MGSA (20). This expression is also shown to be strongly induced by factors involved in inflammation and to exhibit neutrophil chemotactic activity (4). An increase in vascularity is associated with tumor progression in melanocytic lesions from benign melanocytic nevi to MM (21). MGSA is suggested not only to affect melanocytic growth (autocrine) but also to facilitate tumor growth by stimulating angiogenesis (paracrine) that can be inhibited with antibodies to MGSA $(5,6)$. Proliferative activity, assessed by Ki-67 monoclonal antibody, has a strong direct correlation with the number of small vessels at the base of melanocytic skin tumors (22). High mean vessel counts are predictive of metastasis and death in thin $(\leq 0.75 \mathrm{~mm}) \mathrm{MM}$ (23). As the recruitment of neutrophils and vascular changes alter the local environment, the nevus may regress over time, or with secondary insults, progression may occur, generating a primary MM with distinct nuclear and cytologic changes. A direct correlation between the quantity of MGSA and the number of chromosomal abnormalities in cultures of benign nevi, newly established melanoma cultures, and in melanoma cell lines exists (24). Development of cytogenetic abnormalities, together with proliferation, is shown in the progression from nevi to MM (25-28). Subsequent tertiary events occur as 
a result of complex changes in growth factor production and response, nuclear events, and/or extracellular matrix, ultimately resulting in progression to a metastatic melanoma (2).

Our data confirm the potential role of MGSA as a local growth factor in proliferation of nevomelanocytes and, to a lesser extent, of dermal melanocytes in MM. It does not appear to be related to progression of MM (depth of invasion or metastatic potential) and hence has no predictive or prognostic value.

Acknowledgments: The authors thank Judy Dunbar for her secretarial assistance and M. Schottroff, M.T., and R. Santoianni, H.T.L., for photographic expertise.

\section{REFERENCES}

1. Bordoni R, Fine R, Murray D, Richmond A. Characterization of the role of melanoma growth stimulatory activity (MGSA) in the growth of normal melanocytes, nevocytes and malignant melanocytes. J Cell Biochem 1990;44:207-19.

2. Richmond A. The pathogenic role of growth factors in melanoma. Semin Dermatol 1991;10:246-55.

3. Krasagakis K, Garbe C, Orfanos CE. Cytokines in human melanoma cells: synthesis, autocrine stimulation and regulatory functions_an overview. Melanoma Res 1993;3:42533.

4. Derynck R, Balentien E, Han JH, Thomas G, Wen D, Samantha AK, et al. Recombinant expression, biochemical characterization, and biological activities of the human MGSA/gro protein. Biochemistry 1990;29:10225-33.

5. Haghnegahdar H, Du J, Wang D, Strieter RM, Burdick MD, Nanney LB, et al. The tumorigenic and angiogenic effects of MGSA/GRO proteins in melanoma. J Leukoc Biol 2000;67: $53-62$.

6. Owen JD, Strieter R, Burdick M, Haghnegahdar H, Nanney L, Shattuck-Brandt R, et al. Enhanced tumor-forming capacity for immortalized melanocytes expressing melanoma growth stimulatory activity/growth-regulated cytokine beta and gamma proteins. Int J Cancer 1997;73:94-103.

7. Hayashi S, Kurdowska A, Cohen AB, Stevens MD, Fujisawa N, Miller EJ. A synthetic peptide inhibitor for alphachemokines inhibits the growth of melanoma cell lines. J Clin Invest 1997;99:2581-7.

8. Richmond A, Thomas HG. Purification of melanoma growth stimulatory activity. J Cell Physiol 1986;129:375-84.

9. Balentien E, Mufson BE, Shattuck RL, Derynck R, Richmond A. Effects of MGSA/GRO alpha on melanocyte transformation. Oncogene 1991;6:1115-24.

10. Wang D, Yang W, Du J, Devalaraja MN, Liang P, Matsumoto $\mathrm{K}$, et al. MGSA/GRO-mediated melanocyte transformation involves induction of RAS expression. Oncogene 2000;19: 4647-59.

11. Kath R, Rodeck U, Menssen HD, Manoianti M-L, Linnenbach AJ, Elder DE, et al. Tumor progression in the human melanocytic system. Anticancer Res 1989;9:865-72.
12. Herlyn M, Clark WH, Rodeck U, Mancianti ML, Jambrosic J, Koprowski H. Biology of tumor progression in human melanocytes. Lab Invest 1987;56:461-74.

13. Perez RP, Zhang P, Bossrhoff A-K, Buettner R, Abu-Hadid M. Expression of melanoma inhibitory activity in melanoma and nonmelanoma tissue specimens. Hum Pathol 2000;31: 1381-8.

14. Tettelbach W, Nanney L, Ellis D, King L, Richmond A. Localization of MGSA/GRO protein in cutaneous lesions. J Cutan Pathol 1993;20:259-66.

15. Richmond A, Thomas HG. Melanoma growth stimulatory activity: isolation from human melanoma tumors and characterization of tissue distribution. J Cell Biochem 1988;36: 185-98.

16. Tokuda Y, Saida T, Mukai K, Takasaki Y. Growth dynamics of acquired melanocytic nevi. Higher reactivity of proliferating cell nuclear antigen in junctional and compound nevi than intradermal nevi. J Am Acad Dermatol 1994;31:220-4.

17. Kanter L, Blegen H, Wejde J, Lagerlof B, Larsson O. Utility of a proliferation marker in distinguishing between benign naevocellular naevi and naevocellular naevus-like lesions with malignant properties. Melanoma Res 1995;5:345-50.

18. Rudolph P, Lappe T, Schubert C, Schmidt D, Parwaresch RM, Christophers E. Diagnostic assessment of two novel proliferation-specific antigens in benign and malignant melanocytic lesions. Am J Pathol 1995;147:1615-25.

19. Fleming M, Howe SF, Candel AG. Immunohistochemical localization of cytokines in nevi. Am J Dermatopathol 1992; 14:496-503.

20. Wen D, Rowland A, Derynck R. Expression and secretion of gro/MGSA by stimulated human endothelial cells. EMBO J 1989;8:1761-6.

21. Barnhill RL, Fandrey K, Levy MA, Mihm MC, Hyman B. Angiogenesis and tumor progression of melanoma. Quantification of vascularity in melanocytic nevi and cutaneous malignant melanoma. Lab Invest 1992;67:331-7.

22. Smolle J, Soyer H-P, Hofmann-Wellenhof R, Smolle-Juettner F-M, Kerl H. Vascular architecture of melanocytic skin tumors. A quantitative immunohistochemical study using automated image analysis. Pathol Res Pract 1989;185:740-5.

23. Graham CH, Rivers J, Kerbel RS, Stankiewicz KS, White WL. Extent of vascularization as a prognostic indicator in thin $(<0.76 \mathrm{~mm})$ malignant melanomas. Am J Pathol 1994;145: $510-4$.

24. Richmond A, Fine R, Murray D, Lawson DH, Priest JH. Growth factor and cytogenetic abnormalities in cultured nevi and malignant melanomas. J Invest Dermatol 1986;86: 295-302.

25. Weterman MAJ, Van Muijen GNP, Bloemers HPJ, Ruiter DJ. Molecular markers of melanocytic tumor progressions. Lab Invest 1994;70:593-608.

26. Dooley TP. Recent advances in cutaneous melanoma oncogenesis research. Oncol Res 1994;6:1-9.

27. Bjornhagen V, Bonfoco E, Brahme EM, Lindholm J, Auer G. Morphometric, DNA, and proliferating cell nuclear antigen measurements in benign melanocytic lesions and cutaneous malignant melanoma. Am J Dermatol 1994;16:615-23.

28. Grammatico P, Roccella M, Catricala C, Roccella F, Bucher S, Mordenti C, et al. Involvement of the 4q21 region in human malignant melanomas: cytogenetic and immunocytochemical characterization of three primary cell cultures. World J Surg 1995;19:350-1. 